\title{
The Shapes of Cooperatively Rearranging Regions in Glass Forming Liquids
}

\author{
Jacob D. Stevenson, ${ }^{1}$ Jörg Schmalian, ${ }^{2}$ and Peter G. Wolynes ${ }^{1}$ \\ ${ }^{1}$ Department of Physics and Department of Chemistry and Biochemistry, \\ University of California, San Diego, La Jolla, CA 92093 \\ ${ }^{2}$ Department of Physics and Astronomy and Ames Laboratory, Iowa State University, Ames, IA 50011
}

(Dated: September 27, 2018)

\begin{abstract}
The shapes of cooperatively rearranging regions in glassy liquids change from being compact at low temperatures to fractal or "stringy" as the dynamical crossover temperature from activated to collisional transport is approached from below. We present a quantitative microscopic treatment of this change of morphology within the framework of the random first order transition theory of glasses. We predict a correlation of the ratio of the dynamical crossover temperature to the laboratory glass transition temperature, and the heat capacity discontinuity at the glass transition, $\Delta C_{p}$. The predicted correlation agrees with experimental results for the 21 materials compiled by Novikov and Sokolov.
\end{abstract}

Our increased ability to visualize and experimentally probe supercooled liquids on the nanometer length scale has explicitly revealed the presence of cooperatively rearranging regions 1,2.3.4.5.6.7.8.9.10 (CRR's). The cooperative rearrangement of groups of many molecules has long been thought to underlie the dramatic slowing of liquid dynamics upon cooling and could also explain the non-exponential time dependence of relaxation in glassy liquids. Activated transitions of regions of growing size were postulated in the venerable Adam-Gibbs argument for the glass transition ${ }^{11}$. To move, a region, in the AG view, must have a minimum of two distinct conformational states. Natural as this suggestion is, the sizes predicted from literally applying this notion are far too small to explain laboratory observations. The minimal AG cluster would have only two particles since the measured entropy per particle is of order $1 k_{B}$ at the glass transition.

A distinct approach, the random first order transition (RFOT) theory of glasses, is based on a secure statistical mechanical formulation at the mean field

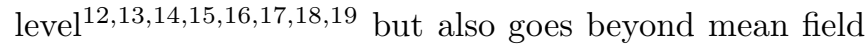
theory to explain the non-exponential, non-Arrhenius dynamics of supercooled liquids through the existence of compact, dynamically reconfiguring regions ("entropic droplets" $)^{20.21 .22}$ whose predicted size is, in contrast to the Adam-Gibbs bound, very much consistent with what has been measured (125-200 molecules), using both scanning microscopy ${ }^{1.8}$ and NMR techniques ${ }^{7.9}$, at temperatures near to $T_{g}$.

Computer simulations ${ }^{3.23 .24}$ and light microscopy studies of colloidal glasses ${ }^{10}$, however, have revealed dynamically reconfiguring regions that are not compact and contain fewer particles. Some investigators describe these regions as "fractal ${ }^{6}$ " while others use the term "strings 3 " to characterize them. It has been suggested that such "stringy" excitations should be taken as the fundamental objects in the theory of glass transitions. As we will show, the fractal nature of the dynamically reconfiguring regions in the relatively high temperature regime probed in current computer simulations follows naturally from RFOT theory. To be precise, RFOT theory predicts the shape of the reconfiguring regions changes from compact to fractal as the system is heated from low temperatures, characteristic of the laboratory glass transitions, to the higher dynamical crossover temperature, $T_{A}$, above which motions are no longer activated. This transformation is shown in figure 10 Owing to the rather short time scale accessible to computer studies, simulations have been inevitably carried out near this dynamical crossover. Likewise, colloidal glasses in the laboratory are studied near to the dynamic crossover because the large size of colloidal particles, in molecular terms, means that the elementary constituents of such suspensions intrinsically move slowly. The dimensionless time scales probed in experiments on colloids are rather similar to those in computer studies.

According to RFOT theory, the dynamical crossover from activated motion has the character of a spinodal ${ }^{17.25}$. Since an analogous change of morphology of nucleation clusters is predicted to occur in ordinary first order transitions ${ }^{26}$, others have already suggested that the dynamically heterogeneities near $T_{A}$ should be fractal or percolation like $e^{27}$. Here we show how the RFOT theory predicts the temperature range where the metamorphosis from compact to fractal happens. We will show that according to RFOT theory, the gap between the dynamical crossover temperature and the glass transition temperature for molecular liquids should correlate inversely with the configurational heat capacity. The existing experimental data confirm this prediction.

The mean field theory of random first order transitions starts by constructing aperiodic minima of a free energy functiona ${ }^{19}$. These aperiodic structures reflect density patterns of molecular units vibrating in the vicinity of their current locations $19,28.29 .30$. These aperiodic free energy minima resemble the so-called "inherent structures" that are minima of the potential energy, i.e. the latter are local minima of the $T=0$ free energy ${ }^{31}$. At finite temperature, these aperiodic structures represent a compromise between the cost of localizing a molecule $S_{l o c}$ and the free energy gain realized by particles being able to get out of each others way once localized. The latter free energy contribution is represented by an interaction term in the 


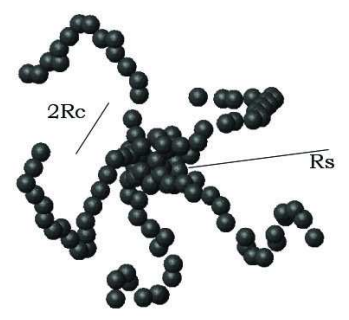

a.

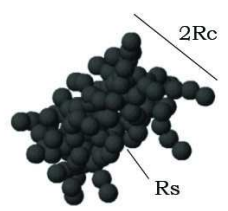

c.

FIG. 1: The schematic appearance of the reconfiguring regions predicted by RFOT theory according to the free energy profiles of the fuzzy sphere model (see text) at: a. the final state near $T_{c}^{\text {string }}$, b. the transition state near $T_{c}^{\text {string }}, \mathbf{c}$. the final state near $T_{g}$, d. the transition state near $T_{g}$. The labeled lines indicate the radii of the core, $R_{c}$, and of the fuzzy halo, $R_{s}$.

usual free energy density functional. Any resulting localized solution is only metastable. The difference of free energy of the typical localized solution and the uniform state is the configurational entropy times the absolute temperature, as confirmed using replica methods 18 .

To estimate the interaction energies, Xia and Wolynes $^{21}$ pointed out that their total, at the Kauzmann temperature $T_{K}$ where the configurational entropy vanishes, must equal the localization cost $T_{K} S_{\text {loc }}$. Therefore, if a typical molecule has z nearest neighbors a local interaction must contribute a term $v_{\text {int }}=(1 / z) T_{K} S_{l o c}$ on the average. The localization entropy cost, in the free energy functional, depends logarithmically on the amount of space each molecule can move in while being encaged: $S_{l o c}=\frac{3}{2} k_{B} \log \left(\alpha_{L} / \pi e\right)$ where $\alpha_{L}$ is the inverse square of the so-called Lindemann ratio of the r.m.s. vibrational amplitude in the glass to the intermolecular spacing. The Lindemann ratio is predicted by detailed microscopic calculations ${ }^{19,30,32}$ and can be measured by neutron scattering where it determines the height of the long-time plateau. The Lindemann ratio only weakly depends on the intermolecular potential and is of order $\frac{1}{10}$ near $T_{g}$. Thus we see, the microscopic RFOT theory suggests $v_{\text {int }}$ should be nearly the same in units of $k_{B} T_{K}$ for all molecular glass formers made of spherical particles and predicts its value. This near universality of the interaction per molecular unit allows the RFOT theory to make quantitative predictions in a wide variety of substances of many measured quantities characterizing glassy motion such as the typical barriers near $T_{g}^{21}$, the degree of nonexponentiality ${ }^{22}$ and the correlation length.

Once the density functional approach has generated aperiodic minima, the dynamics of escape from a given one resembles very much the dynamics of overturning regions of a random field Ising magnet (RFIM) in a field. The free energy difference on a site predicted by the density functional acts like a magnetic field of magnitude $T S_{c}(T)$ in the RFIM. This quantity fluctuates, and the magnitude of its fluctuations are of the order $\sqrt{k_{B} T \Delta C_{p}}$ where $\Delta C_{p}$ is the configurational heat capacity of the fluctuating region. The interaction between a pair of sites in the RFIM analogy is $v_{\text {int }}$, which is already computed. Using this quantitative analogy, RFOT theory computes the typical escape barrier and the fluctuations of the barriers near $T_{K}$.

To estimate the barriers for escape, we can characterize the shape of a reconfiguring region by the number of contiguous sites $\mathrm{N}$ that are rearranged and the number of surface interactions that are broken, b. Near to $T_{K}$, the regions that dynamically reconfigure should be compact because this involves losing the smallest number of favorable interactions, b, while gaining the same configurational entropy proportional to $\mathrm{N}$.

Maximal compactness implies a roughly spherical shape giving a free energy cost

$$
\Delta F(N)=-T S_{c} N+v_{\text {int }} \frac{z}{2} 4 \pi\left(\frac{N}{4 \pi / 3}\right)^{2 / 3}
$$

yielding a barrier that diverges in three dimensions like $S_{c}^{-2}$. This result is modified by the multiplicity of aperiodic states. In analogy to Villain's treatment of the $\mathrm{RFIM}^{33}$, near $T_{K}$ the interface of the reconfigured region between any two aperiodic patterns will be wetted by other specific aperiodic minima that better match the two abutting regions than they do already. This lowers the surface energy term to now scale like $N^{1 / 2}$. This form for the mismatch energy restores the scaling relations near $T_{K}{ }^{20}$. This wetting effect has been shown to be connected with additional replica symmetry breaking in the interface in replica instanton calculations 13.34 . Wetting cannot occur at short ranges so the scale of this mismatch term still follows from $v_{\text {int }}$. In this way the observed Vogel-Fulcher scaling near $T_{K}$ is predicted, $\Delta F^{\ddagger} \propto S_{c}^{-1}$. The numerical proportionality coefficient can be computed from the microscopic value of $v_{i n t}=$ $\frac{1}{z} \frac{3}{2} k_{B} T_{K} \log \frac{\alpha_{L}}{\pi e}$, and the result is a universal multiple of $k_{B} T_{K}$. This resulting prediction of absolute activation barriers agrees well with experimental results for 44 substances 35.36 , a typical deviation being less than $20 \%$.

The compact shape of the CRR and the Vogel-Fulcher behavior are asymptotically correct near $T_{K}$. We now emphasize that away from $T_{K}$ the CRR need not be compact. Other shapes have an entropy advantage: Although the sphere (for which $b=\frac{z}{2} 4 \pi\left(\frac{N}{4 \pi / 3}\right)^{2 / 3}$ ) is unique, there are many contiguous structures with other shapes. Increased temperature favors these more ramified shapes as CRR's. Contiguous shapes are called lattice animals 37 . Much work has gone into enumerating lattice animals because of their importance in problems such as in percolation ${ }^{38}$, Yang-Lee zeros ${ }^{39}$, etc. Klein and 
Unger $^{26}$ emphasized that near a spinodal of an ordinary first order transition the dominant nuclei should be lattice animals characteristic of clusters at the percolation threshold. We now extend this reasoning to random first order transitions.

Accounting for the multiple possible shapes of a CRR we can write the free energy of moving any cluster of $\mathrm{N}$ sites with $b$ boundary interactions as

$$
\Delta F(N, b)=-T S_{c} N+v_{i n t} b-k_{B} T \log (\Omega(N, b))
$$

where $\Omega(N, b)$ is the number of lattice animals of given $\mathrm{N}$ and $\mathrm{b}$. For a given $\mathrm{N}$ the most numerous shapes are percolation-like. When these states dominate we can use enumeration studies near the percolation limit to evaluate $\Omega(N, b)$. In percolation clusters, Leath ${ }^{40}$ determined that for large $\mathrm{N}$,

$$
\Omega_{\text {perc }}(N, t) \sim\left(\frac{(\alpha+1)^{\alpha+1}}{\alpha^{\alpha}}\right)^{N} \exp \left(-\frac{N^{2 \phi}}{2 B^{2}}\left(\alpha-\alpha_{e}\right)^{2}\right)
$$

Here, $\alpha=t / N$, and $t$ is the number of unoccupied sites bounding the occupied cluster. We will take $\phi$ to have its mean field value of $1 / 2$. B is a lattice dependent constant. $B=1.124$ (for the face centered cubic lattice) follows from fitting the Leath formula to numerics calculated by Sykes et al ${ }^{41}$ for $N \leq 9$. The mean value of $t / N$ approaches $\alpha_{e}=\left(1-p_{c}\right) / p_{c}$ for large $\mathrm{N}$ at the percolation threshold, $p_{c}$ ( $p_{c}=0.198^{41}$ for the FCC lattice).

Ideally we would like to evaluate the needed percolation quantities for random close packed lattices. There is an ambiguity here as to the definition of "contact". Clearly spheres need not precisely touch (as in, say, percolation conductivity experiments), but rather their surfaces may be separated by at most a Lindemann length in order to be called connected. While the parameters for this continuum percolation problem are not available, they can be easily estimated since the percolation quantities primarily depend on the near neighbor connectivity. The number of neighbors in the rcp lattice is roughly the same as the face centered cubic (FCC); thus it is reasonable to use parameters for an FCC close packed lattice of spheres.

The number of bonds, b, is related to Leath's t. For the simple cubic lattice, $\frac{\langle b\rangle}{\langle t\rangle}=1.67^{42}$. This ratio is linear in coordination number, $\mathrm{z}$, so we can again find the value for the rcp lattice. Thus, following from equation 2

$$
\Delta F(N, t)=-T S_{c} N+v_{i n t} 1.68 \frac{z}{z_{S C}} t-k_{B} T \log \left(\Omega_{\text {perc }}(N, t)\right)
$$

To find the most probably escape route we need to find the most probable $t$ as a function of $\mathrm{N}$. If we minimize equation 4 with respect to $t$ using equation 3 for $\Omega$, we find that the most probable value of $t$ is $\bar{t}=\bar{\alpha} N$ where $\bar{\alpha}=3.10$. With this most probable value, $\Omega_{\text {perc }}$ becomes simply $\Omega_{\text {perc }}=\lambda^{N}$ where $\lambda=7.64$. Thus each term in equation 4 is now proportional to $\mathrm{N}$, and the free energy becomes.

$$
\Delta F(N)=\left(-T S_{c}+v_{i n t} 1.68 \frac{z_{F C C}}{z_{S C}} \bar{\alpha}-k_{B} T \log (\lambda)\right) N
$$

Apart from $S_{c}$ each term in this expression follows from a microscopic calculation. The nature of the profile therefore depends on the configurational entropy. Clearly the free energy for percolative CRR's either monotonically increases or decreases with $\mathrm{N}$. If the free energy profile increases with $\mathrm{N}$, a reconfiguration event due to a percolation cluster is impossible, so a more compact structure that will eventually become stable for large $\mathrm{N}$ provides the dominant reconfiguration route. If $\mathrm{F}$ decreases with $\mathrm{N}$ for the percolation shape, no barrier at all should be observed. Clearly the change of behavior of $\Delta F(N)$, from increasing to decreasing with $\mathrm{N}$, signals a crossover to non-activated dynamics. Taking $v_{i n t}$ to be entirely entropic and putting the determined RFOT values of $v_{\text {int }}$ into equation 5 yields.

$$
\begin{aligned}
\Delta F(N) & =\left(-T S_{c}+k_{B} T(3.20-1.91)\right) N \\
& =-T\left(S_{c}-k_{B} 1.28\right) N
\end{aligned}
$$

We see barrier-less reconfiguration events occur at a critical configurational entropy, $S_{c}^{\text {perc }}=1.28 k_{B}$ if we neglect the mean field softening effects. Using the thermodynamic relation, $S_{c}(T)=S_{\infty}\left(1-T_{K} / T\right)$ where $S_{\infty}$ is given by $\Delta C_{p}\left(T_{g}\right) T_{g} / T_{K}$, RFOT theory thus predicts the crossover transition temperature, $T_{c}^{\text {perc }}$.

$$
\frac{T_{c}^{\text {perc }}}{T_{K}}=\left(1-\frac{S_{c}^{p e r c}}{\Delta C_{p}} \frac{T_{K}}{T_{g}}\right)^{-1}
$$

The bigger $\Delta C_{p}$ is, the closer $T_{c}^{p e r c}$ will be to $T_{K}$; more "fragile" liquids with larger $\Delta C_{p}$ have a smaller activated range, while a broader range for activated transport applies for stronger liquids with smaller $\Delta C_{p}$. A similar trend is predicted for the mean field crossover based on detailed microscopic calculations for fluids with network network structure by Hall and Wolynes 32 who suggest an entropy at the higher mean field crossover of $S_{c}\left(T_{A}\right)=2.0 k_{B}$. Including the softening of $v_{\text {int }}$ expected as this mean field transition is approached lowers the estimate of the percolation point. The amount of lowering is uncertain, however, because simultaneous with the softening a broadening of the interface is expected, thus effectively reducing the possible entropy gain from shape fluctuations. RFOT theory indicates that at the same configurational entropy level, on the average, the nature of transitions will be the same in fragile and strong liquids. As in the RFOT theory of the non-exponentiality parameter $\beta^{22}$, fluctuations in the driving force depend on $\Delta C_{p}$ explicitly and should be included in equation 1 Thus fast and slow CRR's would have somewhat different shapes (faster being more ramified generally since their entropy is higher). We do not discuss this effect further here, however.

We see that the counting problems for percolation clusters are not all that different from those relevant for 
strings. We can re-do the crossover transition argument for purely string-like objects. The number of broken interactions of a string scales with length, $N(z-2)$, as does the shape entropy of a string, $\log (\Omega)=N \log (z-5)$. $(z-5)$ represents the number of directions a string can take that excludes backtracking on top of, or directly next to, the previous particle. The next neighbor position is disallowed because, if occupied, the cluster becomes compact. Putting these coefficients into the free energy of growing a string of length $\mathrm{N}$, we find that string growth will become the down hill route of escaping a minimum at an entropy of $S_{c}^{\text {string }}=1.13 k_{B}$. This results in a crossover temperature

$$
\frac{T_{c}^{\text {string }}}{T_{K}}=\left(1-\frac{S_{c}^{\text {string }}}{\Delta C_{p}} \frac{T_{K}}{T_{g}}\right)^{-1}
$$

a bit lower than predicted by percolation. In Figure 2 a we plot the predicted $T_{c}^{\text {string }}$ and $T_{c}^{\text {perc }}$ versus $1 / \Delta C_{p}$ for various liquids. Crossover temperatures from activated to non-activated dynamics have been determined by Stickel plot analysis ${ }^{43}$. Experimental crossover temperatures for 21 substances obtained in this way by Novikov and Sokolov $\underline{44}$ are also plotted in the figure. Some of the outliers are polymers for which other slowing effects compound simple RFOT results(). Uncertainty in the determination of $T_{K}$ for very strong liquids probably is a source of the discrepancy between the theory and experiment for these latter substances. We have also plotted $\left(T_{c}-T_{g}\right) / T_{g}$ in figure 2$]$. According to RFOT theory the entropy at $T_{g}$ is $S_{c}\left(T_{g}\right)=\Delta C_{p}\left(T_{g}-T_{K}\right) / T_{K}=0.79 k_{B}$. The value of $S_{c}\left(T_{g}\right)=0.79 k_{B}$ gives a glass transition occurring at $10^{10} \mathrm{P}$, as was used in the experimental papers (see figure 4). The quantitative agreement of the experimental crossover temperatures and the present predictions of the string and percolation transitions is striking.

To predict quantitatively the barriers and the typical shapes of reconfiguring regions at temperatures between $T_{c}$ and $T_{K}$ we must find a suitable analytic form of $\Omega(N, b)$ for all relevant values of $\mathrm{N}$ and b. Surface roughening theories give predictions of $\Omega(N, b)$ valid only for nearly spherical objects ${ }^{45}$, and would be useful only near $T_{K}$. The percolation theory gives an explicit form of $\Omega$ valid only for the most populous ramified, fractal shapes that dominate near the crossover.

We use a reasonably effective, but unabashedly approximate, treatment of the animal counting problem to interpolate between these limits. We describe the reconfiguring region as a "fuzzy sphere," an object with a spherical core of $n_{c}$ particles, but surrounded by a ramified, but connected, halo of $n_{f}$ particles. If we let the core size, $n_{c}$, go to zero we are left with only an extended object. Conversely, if we let the halo vanish then we have only a sphere. We would like to describe the halo as a percolation cluster, but all the relevant geometric quantities are not precisely known. We therefore model the halo as a set of strings of particles extending from the surface of the central core. In this way we can explicitly determine the entropic contribution of a halo of $n_{s}$ strings.
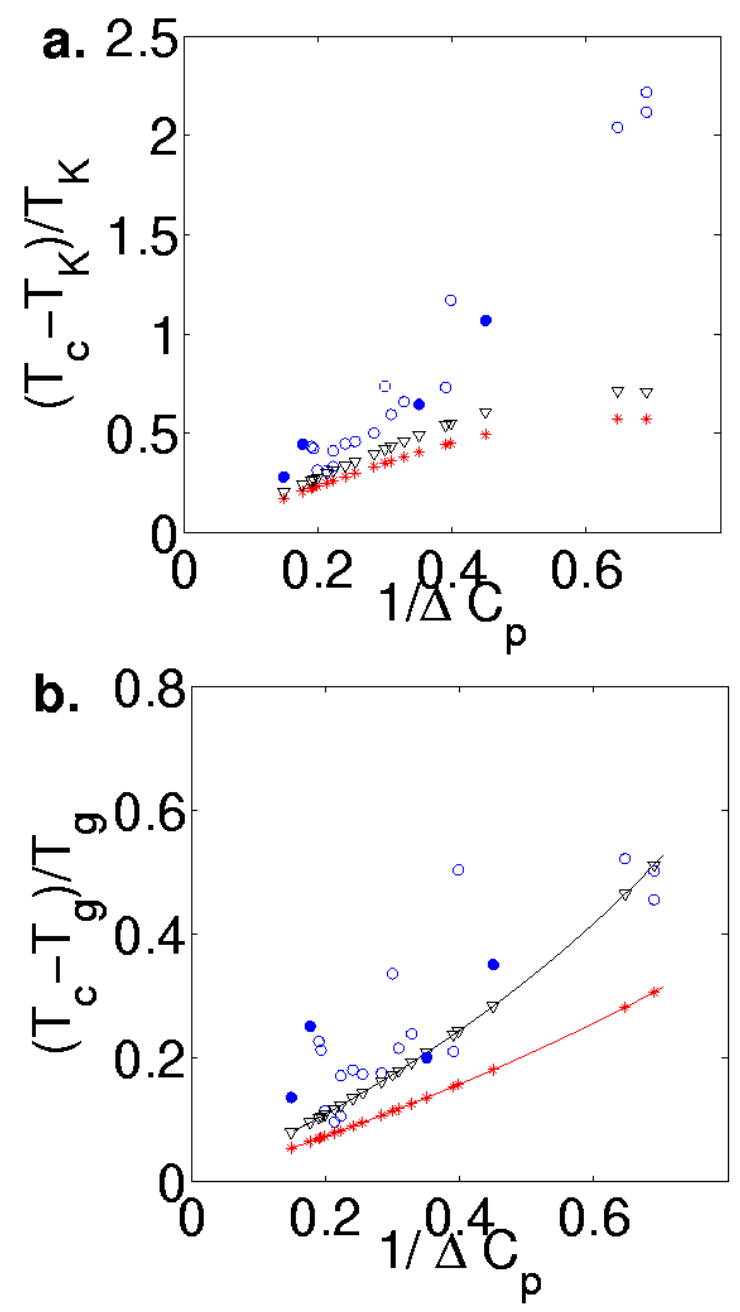

FIG. 2: a. Predictions for $\left(T_{c}^{\text {string }}-T_{K}\right) / T_{K}$ (depicted as $\left.*_{\text {stars }}\right)$ and $\left(T_{c}^{\text {perc }}-T_{K}\right) / T_{K}$ (depicted as triangles). The experimentally derived crossover temperatures, $\left(T_{c}^{e x p}-T_{K}\right) / T_{K}$, from those materials collected by Novikov and Sokolov ${ }^{44}$, are shown as circles with the dark circles referring to polymers. In all cases the values for the Kauzmann temperature, $T_{K}$, were taken from the correlation $T_{K}=T_{g}(1-16 / m)$ found in a paper by Böhmer and Angell ${ }^{46}$. b. Same as for a. except a plot of $\left(T_{c}-T_{g}\right) / T_{g}$ instead of $\left(T_{c}-T_{K}\right) / T_{K}$. The conversion ratio $T_{K} / T_{g}$ was set through $S_{c}\left(T_{g}\right)=\Delta C_{p}\left(T_{g}-T_{K}\right) / T_{K}=$ $0.79 k_{B}$. For both plots the $\Delta C_{p}$ values for the materials were determined from their $\mathrm{m}$ values through the correlation $m=20.7 \Delta C_{p}$ discussed in Stevenson and Wolynes ${ }^{36}$, where $\Delta C_{p}$ is, from RFOT theory, the heat capacity discontinuity at $T_{g}$ per independently moving unit, or "bead."

For $S_{c}$ between the extremes of $0\left(\right.$ at $T_{K}$ ) and $S_{c}^{\text {string }}$ we can now compute the full activation free energy profile for CRR's pictured as fuzzy spheres. 


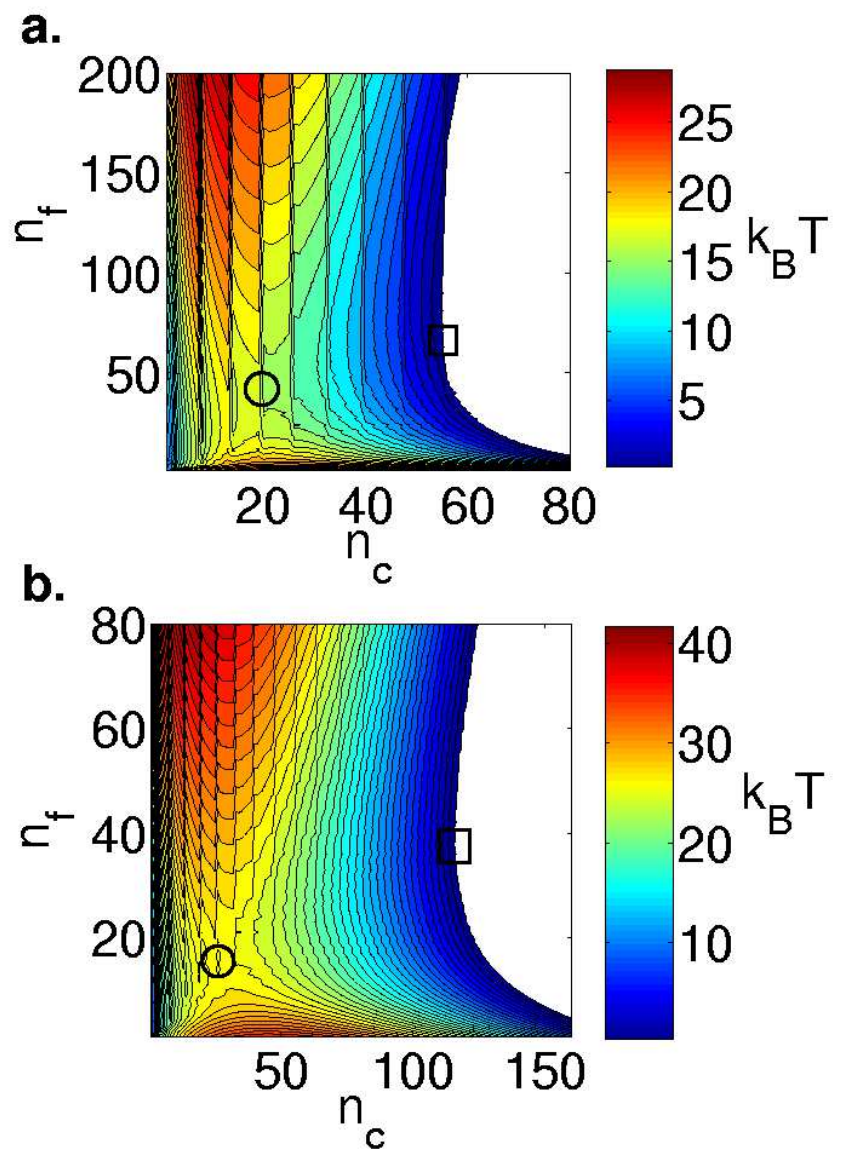

FIG. 3: Two dimensional free energy profiles as functions of the number of sites in the core, $n_{c}$, and the number in the fuzzy halo, $n_{f}$, a. near $T_{c}^{\text {string }}$ and $\mathbf{b}$. near $T_{g}$. The sidebar is in units of $k_{B} T$ with the contours lines corresponding to intervals of $1 k_{B} T$. The circles indicate the location of the typical transition state. The squares indicate a fully reconfigured region.

$$
\begin{gathered}
\Delta F\left(n_{c}, n_{f}, n_{s}\right)=v_{\text {int }} \frac{z}{2}\left(\frac{4 \pi / 3}{n_{c}}\right)^{1 / 6}\left(4 \pi\left(\frac{n_{c}}{4 \pi / 3}\right)^{2 / 3}-n_{s}\right)+v_{\text {int }}(z-2) n_{f} \\
-T S_{c}\left(n_{c}+n_{f}\right)-k_{B} T \log \left(\Omega\left(n_{c}, n_{f}, n_{s}\right)\right) \\
\Delta F\left(n_{c}, n_{f}\right)=-\log \left(\sum_{n_{s}} \exp \left(-\Delta F\left(n_{c}, n_{f}, n_{s}\right)\right)\right)
\end{gathered}
$$

The full expression for the shape entropy of a fuzzy sphere, $\Omega$, is found in the supplementary material. This estimate accounts for the excluded volume between the strings via Flory theory ${ }^{50}$. Figures 3 a and 3 b show contour plots of the free energy at a configurational entropy value near the dynamic crossover and near the glass tran- sition respectively. The saddle points on these free energy surfaces describe transition state ensembles for activated reconfiguration events. The resulting barrier depends universally on the configurational entropy and is plotted as a function of $S_{c}\left(T_{g}\right) / S_{c}(T)$ in figure 4 Superimposed on the graph are the experimental viscosity barrier 


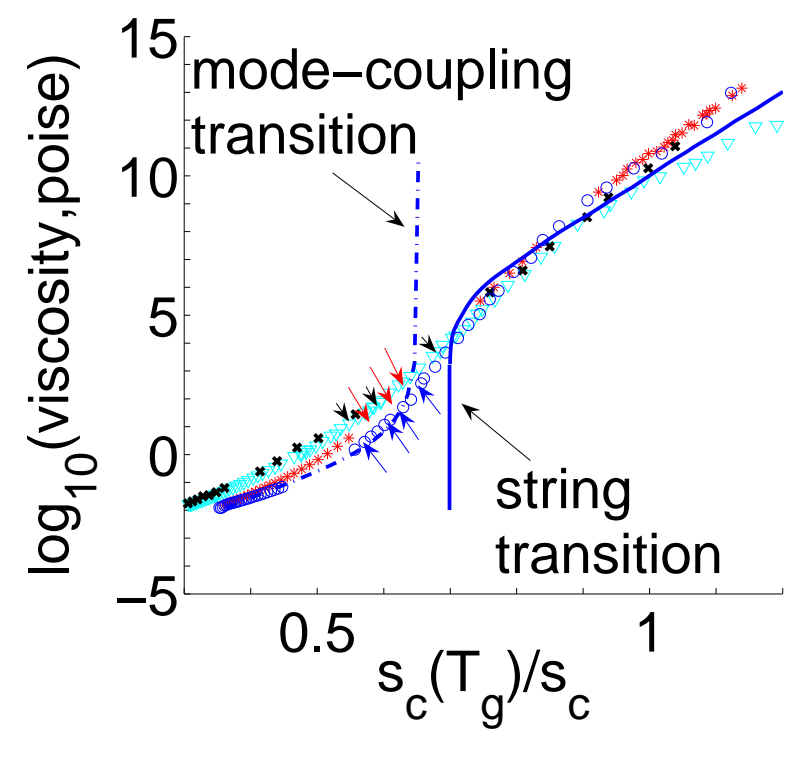

FIG. 4: A comparison of experimental viscosity barriers with the barriers predicted from the fuzzy sphere model (solid line). Salol data ${ }^{43}$ are represented with circles, Propylene Carbonate data ${ }^{43}$ with crosses, O-Terphenyl data ${ }^{47.48}$ with stars, and alpha-Phenyl-O-Cresol data ${ }^{47,48}$ with triangles. An experimental mode coupling fit to salol (Hinze et al. ${ }^{49}$ ) is shown with a dot-dashed line. Experimentally derived values of the entropy at the crossover transitions 44 are shown with arrows. The free energy barriers were placed on the $\log _{10}$ (viscosity) curve by setting $\Delta F^{\ddagger}=0$ to correspond with the large T experimental value of 1 centipoise for the viscosity. At the measured calorimetric glass transition, corresponding to a cooling rate of $10^{\circ} / \mathrm{min}$, the viscosity of the materials is about $10^{10} P$. This value was used to determine the theoretical value $S_{c}\left(T_{g}\right)=0.79 k_{B}$.

for several glass forming liquids of varying fragility and of known entropy and heat capacity. The universality is clearly confirmed (In these plots the calorimetrically determined $T_{g}$ 's were used for calibration, not the viscometric values!). The barrier clearly depends linearly on $1 / S_{c}$ for $S_{c}<S_{c}^{\text {string }}$ consistent with the asymptotic RFOT analysis, but as the critical value of the configurational entropy, $S_{c}^{\text {string }}$, is approached the activation barrier rapidly decreases, dropping to zero at $S_{c}^{\text {string }}$. We have also included in the plot the experimental mode coupling fit to the viscosity for salol 19 . The symmetry is striking; the mode coupling theory fits the dynamic transition from above, while the current argument predicts its emergence from below.

As well as giving the barriers, this calculation suggests the dominant routes the liquid takes to reconfigure and the final form of the CRR's in different entropy regimes. The final states are broadly distributed in shape as shown via the broad $1 k_{B} T$ contour in the plots. We define the characteristic final shape as the one with the smallest

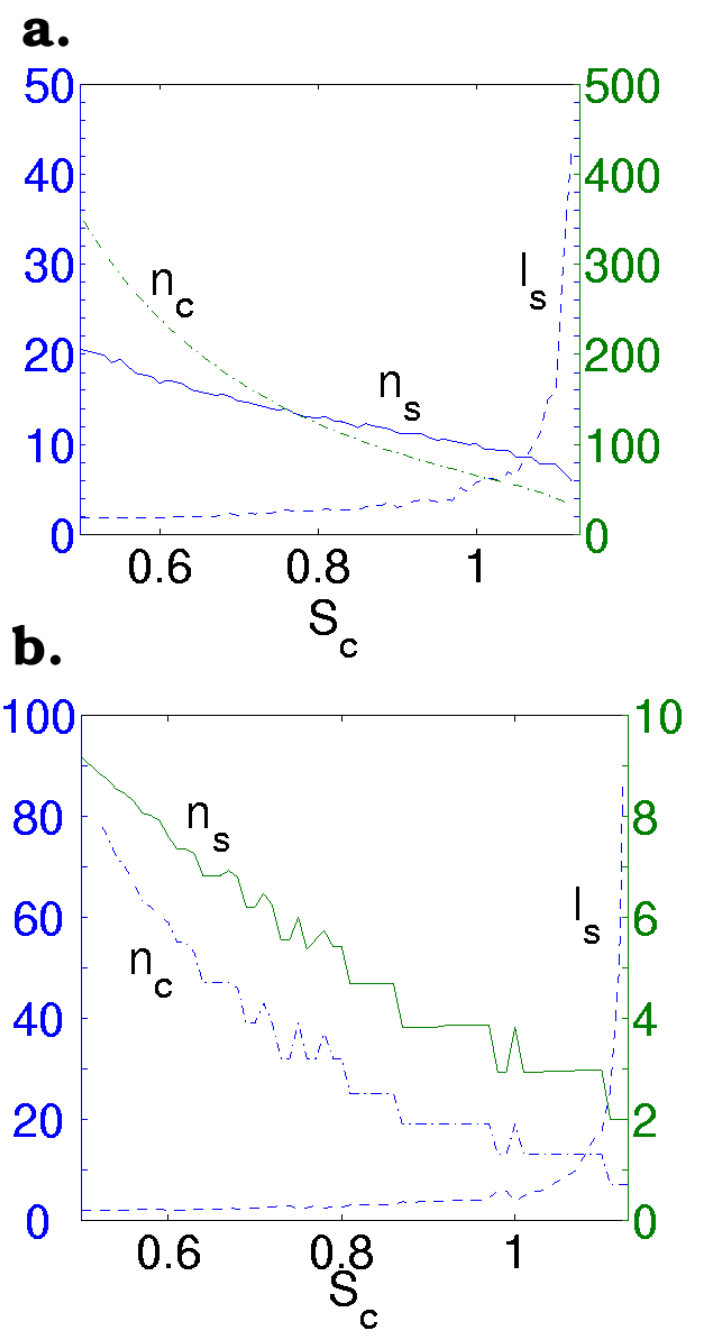

FIG. 5: Predictions for the fuzzy sphere shape characteristics are shown as functions of the configurational entropy for the final state and the transition state. $n_{c}$ (dashed-dotted line) is the number of particles in the core, $n_{s}$ (solid line) is the number of strings and $l_{s}$ (dashed line) is the typical length of a string. a. The final state: $n_{c}$ (dashed-dotted line) uses the axes on the right while $n_{s}$ (solid line) and $l_{s}$ (dashed line) use the axes on the left. b. The transition state: here $n_{s}$ (solid line) uses the axes on the right while $n_{c}$ (dashed-dotted line) and $l_{s}$ (dashed line) use the axes on the left. The sizes and lengths are given in terms of the number of particles. The strings in the halo are nearly random flight chains giving the physical dimensions in figure 6

core. We show examples of the final shape expected near $T_{g}$ and near the crossover temperature in figure 1 Figures 5 and 6 show how the characteristic scales of the transition states and final reconfigured regions change with configurational entropy. Near $T_{g}$ the shapes are mostly spherical with just a small fraction of the particles in the stringy halo and have sizes consistent with the previous XW estimate. A typical protuberance on the compact core near $T_{g}$ is only 2 particles long. Near 


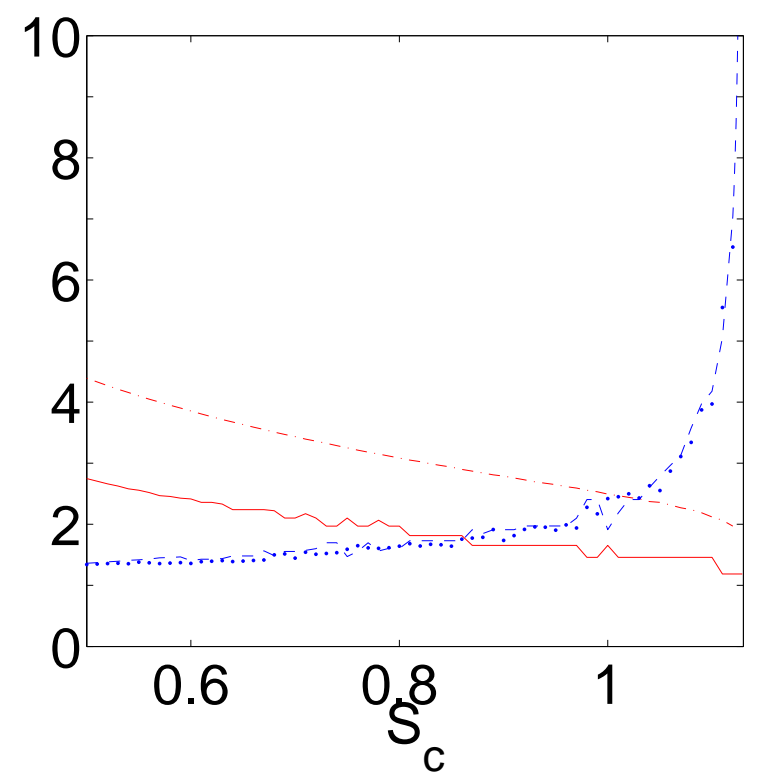

FIG. 6: The radius of the core, $R_{c}$, at the transition state (solid line) and at the final state (dashed-dotted line). Also, the radius of the stringy halo, $R_{s}$, at the transition state (dashed line) and the final state (dotted line). The radii are given in terms of the number of particles.

$T_{c}^{\text {string }}$, however, the core size becomes very small while the strings lengthen dramatically. This growth occurs for both the transition state and the final state.

The string lengths near $T_{c}^{\text {string }}$ are larger than what is usually reported in simulation or in microscopy studies. This apparent discrepancy arises from a kinetic effect which we describe as follows: Though the free energy barrier for creating a string approaches zero at $T_{c}^{\text {string }}$, the actual time to construct a string not only remains finite but indeed grows with the length of the string. The barrier to create a new string is somewhat larger than to extend an old one. Because of this, the growth/death of a string, generally takes place particle by particle on the microscopic time scale, and should be a diffusive process, with growth time $\tau_{s}=\tau_{\text {micro }}^{0} l_{s}^{2}$. Here, $\tau_{\text {micro }}^{0}$ is a typical vibrational time scale, i.e.the time for a particle to explore its cage. When $\tau_{s}$ becomes comparable to the time for another activated event to occur in the immediate vicinity of the string, $\tau_{\alpha} / l_{s}$, the growth of the original string will be perceived to have been interrupted. Here, $\tau_{\alpha}=\tau_{\text {micro }}^{0} e^{F^{\ddagger} / k_{B} T}$. This finite growth time gives a maximum limit for the length of strings:

$$
l_{s, \max }^{3}=e^{F^{\ddagger} / k_{B} T} .
$$

Larger strings will be interrupted, or "incoherent," since an activated event occurs along the string. Using the fuzzy sphere model, the minimum barrier corresponds to a core region of size 7 . This gives an $F^{\ddagger}$ consistent with what Novikov and Sokolov 44 call the "magic" relaxation time for the crossover and a length $l_{\text {s.max }} \cong e^{14 / 3} \cong 108$. This is larger than the lengths usually quoted from simulations currently made on long time scales, but the rapid variation of $F^{\ddagger}$ and $l_{s}$ near the string transition makes this result rather sensitive to modeling details. Important is that there is a natural cut off length of kinetic origin that causes $T_{c}^{\text {string }}$ to be a crossover scale and not a sharp transition.

We see that the random first order transition theory predicts the CRR's in glassy liquids are compact, nearly spherical objects in the deep supercooled region, but that in the moderately supercooled region, near the mode coupling transition, the CRR's are predicted to become non-compact, extended string-like objects. The crossover temperature is entropically controlled. This prediction of RFOT theory is confirmed by experiment.

\section{Acknowledgments}

Work at UCSD was supported by NSF grant CHE0317017. J. Schmalian was supported by the Ames Laboratory, operated for the U.S. Department of Energy by Iowa State University under Contract No. W-7405Eng-82 (J.S.).

Supplementary Material: The number of ways a fuzzy sphere with $n_{c}$ particles in the central core, $n_{f}$ particles in the stringy halo, and $n_{s}$ strings can be arranged is $\Omega\left(n_{c}, n_{f}, n_{s}\right)$. The entropy, $\log (\Omega)$, of a fuzzy sphere can be broken up into three components

$$
\Omega\left(n_{c}, n_{f}, n_{s}\right)=e^{S_{\text {pos }}+S_{\text {dis }}+S_{\text {grow }}}
$$

$S_{d i s}$ is the number of ways one can distribute $n_{f}$ particles in $n_{s}$ strings ensuring each string has at least one particle.

$$
e^{S_{d i s}}=\frac{\left(n_{f}-1\right) !}{\left(n_{f}-n_{s}\right) !\left(n_{s}-1\right) !}
$$

The positional entropy, $S_{\text {pos }}$, accounts for the number of ways you can position $n_{s}$ strings on a surface of area $n_{\text {surf }}=4 \pi\left(\frac{n_{c}}{4 \pi / 3}\right)^{2 / 3}$. It is necessary to account for the excluded volume of the strings by noting that a string not only excludes its own position, but also $\tilde{z}=z / 2$ of its nearest neighbors as well. Each string placed on the surface further reduces the number of available positions by $\tilde{z}+1$. Using this we can find a form of $S_{\text {pos }}$ by adding particles one at a time. 


$$
e^{S_{\text {pos }}}=\frac{1}{n_{s} !}\left(n_{\text {surf }}\right)\left(n_{\text {surf }}-(\tilde{z}+1)\right)\left(n_{\text {surf }}-2(\tilde{z}+1)\right) \cdots\left(n_{\text {surf }}-\left(n_{s}-1\right)(\tilde{z}+1)\right)
$$

The growth entropy $S_{\text {grow }}$ accounts for the number of configurations the strings can take on as they lengthen. We calculate this excluded volume effect on the entropy of the strings using Flory's method ${ }^{50}$. We imagine the fuzzy halo as made up of $n_{f} / n_{s}$ concentric shells each containing one particle for each of the $n_{S}$ strings. If we place particles one at a time, filling up each shell before starting a new one, then $f_{i j}$ is the expectation value that a given cell is occupied or part of the excluded volume of the $i-1$ previously placed particles in the $j^{\text {th }}$ shell. $f_{i j}$ can be estimated by the fraction of the current shell taken up by the excluded volume of the other particles.

$$
f_{i j}=\frac{\tilde{z}(j-1)}{4 \pi\left(\left(\frac{n_{c}}{4 \pi / 3}\right)^{1 / 3}+i-1\right)^{2}}
$$

Thus, including the $n_{s}$ particles per shell in all $n_{f} / n_{s}$ shells we get

$$
e^{s_{\text {grow }}}=(z-5)^{n_{f}-n_{s}} \prod_{i=2}^{n_{f} / n_{s}} \prod_{j=1}^{n_{s}}\left(1-f_{i j}\right)
$$

${ }^{1}$ Deschenes, L. A. \& Bout, D. A. V. Single-molecule studies of heterogeneous dynamics in polymer melts near the glass transition. Science 292, 255-258 (2001).

2 Ediger, M. D. Spatially heterogeneous dynamics in supercooled liquids. Ann. Rev. Phys. Chem. 51, 99 (2000).

${ }^{3}$ Gebremichael, Y., Vogel, M. \& Glotzer, S. C. Particle dynamics and the development of string-like motion in a simulated monoatomic supercooled liquid. J. Chem. Phys. 120, 4415-4427 (2004).

${ }^{4}$ Kob, W., Donati, C., Plimpton, S. J., Poole, P. H. \& Glotzer, S. C. Dynamical heterogeneities in a supercooled lennard-jones liquid. Phys. Rev. Lett. 79, 2827-2830 (1997).

5 Lacevic, N. \& Glotzer, S. C. Dynamical heterogeneity and jamming in glass-forming liquids. J. Phys. Chem B 108, 19623-19633 (2004).

6 Reinsberg, S. A., Heuer, A., Doliwa, B., Zimmermann, H. \& Spiess, H. W. Comparative study of the nmr length scale of dynamic heterogeneities of three different glass formers. J. Non-Cryst. Solids 307-310, 208-214 (2002).

7 Richert, R. Heterogeneous dynamics in liquids: fluctuations in space and time. J. Phys. Cond. Matt. 24, R703R738 (2002).

${ }^{8}$ Russell, E. V. \& Israeloff, N. E. Direct observation of molecular cooperativity near the glass transition. Nature 408, 695-698 (2000).

9 Sillescu, H. Heterogeneity at the glass transition: a review. J. Non-Cryst. Solids 243, 81-108 (1999).

10 Weeks, E. R., Crocker, J. C., Levitt, A. C., Schofield, A. \& Weitz, D. A. Three-dimensional direct imaging of structural relaxation near the colloidal glass transition. Science 287, 627-631 (2000).

11 Adam, G. \& Gibbs, J. H. On the temperature dependence of cooperative relaxation properties in glass-forming liquids. J. Chem. Phys. 43, 139 (1943).

12 Bouchaud, J. P. \& Biroli, G. On the adam-gibbskirkpatrick-thirumalai-wolynes scenario for the viscosity increase in glasses. J. Chem. Phys. 121, 7347-7354 (2004).

13 Franz, S. Metastable states, relaxation times and freeenergy barriers in finite dimensional glassy systems. arXiv cond-mat/0507585 (2005).

14 Franz, S. \& Toninelli, F. L. A field-theoretical approach to the spin glass transition: models with long but finite interaction range. J. Stat. Mech: Thoer. and Exp. $\mathbf{P 0 1 0 0 8}$ (2005).

15 Kirkpatrick, T. R. \& Thirumalai, D. Dynamics of the structural glass-transition and the p-spin-interaction spinglass model. Phys. Rev. Lett. 58, 2091-2094 (1987).

16 Kirkpatrick, T. R. \& Wolynes, P. G. Connections between some kinetic and equilibrium theories of the glass transition. Phys. Rev. A 35, 3072 (1987).

17 Kirkpatrick, T. R. \& Wolynes, P. G. Stable and metastable states in mean-field potts and structural glasses. Phys. Rev. B 36, 8552 (1987).

18 Mezard, M. \& Parisi, G. Thermodynamics of glasses: A first principles computation. Phys. Rev. Lett. 82, 747 (1999).

19 Singh, Y., Stoessel, J. P. \& Wolynes, P. G. Hard-sphere glass and the density-functional theory of aperiodic crystals. Phys. Rev. Lett. 54, 1059 (1985).

${ }^{20}$ Kirkpatrick, T. R., Thirumalai, D. \& Wolynes, P. G. Scaling concepts for the dynamics of viscous liquids near an ideal glassy state. Phys. Rev. A 40, 1045 (1989).

21 Xia, X. Y. \& Wolynes, P. G. Fragilities of liquids predicted from the random first order transition theory of glasses. Proc. Natl. Acad. Sci. 97, 2990 (2000).

22 Xia, X. Y. \& Wolynes, P. G. Microscopic theory of heterogeneity and nonexponential relaxations in supercooled liquids. Phys. Rev. Lett. 86, 5526 (2001).

23 Donati, C. et al. Stringlike cooperative motion in a supercooled liquid. Phys. Rev. Lett. 80, 2338-2341 (1998).

24 Donati, C., Glotzer, S. C., Poole, P. H., Kob, W. \& Plimpton, S. J. Spatial correlations of mobility and immobility in a glass-forming lennard-jones liquid. Phys. Rev. E 60, 
3107-3119 (1999)

${ }^{25}$ Biroli, G. \& Bouchaud, J. P. Diverging length scale and upper critical dimension in the mode-coupling theory of the glass transition. Europhys. Lett. 67, 21-27 (2004).

${ }^{26}$ Unger, C. \& Klein, W. Nucleation theory near the classical spinodal. Phys. Rev. B 29, 2698-2708 (1984).

27 Johnson, G., Mel'cuk, A. I., Gould, H., Klein, W. \& Mountain, R. D. Molecular-dynamics study of long-lived structures in a fragile glass-forming liquid. Phys. Rev. E 57, 5707-5718 (1998).

${ }^{28}$ Dasgupta, C. \& Valls, O. T. Free energy landscape of a dense hard-sphere system. Phys. Rev. E 59, 3123-3134 (1999).

${ }^{29}$ Fuchizaki, K. \& Kawasaki, K. Dynamical density functional theory for glassy behaviour. J. Phys. - Cond. Matt. 14, 12203 (2002).

30 Stoessel, J. P. \& Wolynes, P. G. Linear excitations and the stability of the hard-sphere glass. J.Chem. Phys. 80, 4502-4512 (1984).

31 Stillinger, F. H. \& Weber, T. A. Dynamics of structural transitions in liquids. Phys. Rev. A 28, 2408-2416 (1983).

${ }^{32}$ Hall, R. W. \& Wolynes, P. G. Microscopic theory of network glasses. Phys. Rev. Lett. 90, 085505 (2003).

${ }^{33}$ Villain, J. Equilibrium critical properties of random field systems - new conjectures. J. Physique 46, 1843-1852 (1985).

34 Dzero, M., Schmalian, J. \& Wolynes, P. G. Activated events in glasses: the structure of entropic droplets. arXiv: cond. matt. 0502011 (2008).

${ }^{35}$ Lubchenko, V. \& Wolynes, P. G. Barrier softening near the onset of nonactivated transport in supercooled liquids: Implications for establishing detailed connection between thermodynamic and kinetic anomalies in supercooled liquids. J. Chem. Phys. 119, 9088-9105 (2003).

${ }^{36}$ Stevenson, J. D. \& Wolynes, P. G. Thermodynamic-kinetic correlations in supercooled liquids: A critical survey of experimental data and predictions of the random first-order transition theory of glasses. J. Phys. Chem. B 109, 1509315097 (2005)

37 Stauffer, D. Monte-carlo study of density profile, radius, and perimeter for percolation clusters and lattice animals.
Phys. Rev. Lett. 41, 1333-1336 (1978).

38 Stauffer, D. Introduction to Percolation Theory (Taylor and Francis Ltd., 1985).

39 Yang, C. N. \& Lee, T. D. Statistical theory of equations of state and phase transitions. i. theory of condensation. Phys. Rev. 87, 404 (1952).

${ }^{40}$ Leath, P. L. Cluster size and boundary distribution near percolation threshold. Phys. Rev. B 14, 5046 (1976).

${ }^{41}$ Sykes, M. F., Gaunt, D. S. \& Glen, M. Percolation processes in three dimensions. J. Phys. A: Math. Gen. 9, 1705 (1976).

${ }^{42}$ Cao, Q. Z. \& Wong, P. O. External surface of site percolation clusters in three dimensions. J. Phys. A: Math. Gen. 25, L69 (1992).

${ }^{43}$ Stickel, F., Fischer, E. W. \& Richert, R. Dynamics of glass-forming liquids .2. detailed comparison of dielectric relaxation, dc-conductivity, and viscosity data. J. Chem. Phys. 104, 2043-2055 (1996).

${ }^{44}$ Novikov, V. N. \& Sokolov, A. P. Universality of the dynamic crossover in glass-forming liquids: A "magic" relaxation time. Phys. Rev. E 67, 031507 (2003).

${ }^{45}$ Chui, S. T. \& Weeks, J. D. Phase-transition in 2dimensional coulomb gas, and interfacial roughening transition. Phys. Rev. B 14, 4976-4982 (1976).

${ }^{46}$ Böhmer, R. \& Angell, C. A. Correlations of the nonexponentiality and state dependence of mechanical relaxations with bond connectivity in Ge-As-Se supercooled liquids. Phys. Rev. B 45, 10091-10094 (1992).

${ }^{47}$ Cukierman, M., Lane, J. W. \& Uhlmann, D. R. Hightemperature flow behavior of glass-forming liquids: A freevolume interpretation. J. Chem. Phys. 59, 3639-3644 (1973).

48 Laughlin, W. T. \& Uhlmann, D. R. Viscous flow in simple organic liquids. J. Phys. Chem 76, 2317-2325 (1972).

${ }^{49}$ Hinze, G., Brace, D. D., Gottke, S. D. \& Fayer, M. D. A detailed test of mode-coupling theory on all time scales: Time domain studies of structural relaxation in a supercooled liquid. J. Chem. Phys. 113, 3723 (2000).

${ }^{50}$ Flory, P. J. Principles of Polymer Chemistry (Cornell University Press Ltd., 1953). 